\title{
Can biosensors detect contaminants in aqueous medium by impedance spectroscopy?
}

\begin{abstract}
Biosensors are powerful tools for environmental, human and animal health monitoring. The development of portable devices based on electrical impedance spectroscopy together with specific biosensors may allow the realization of in situ measurements to determine contaminants in water. As a result, quick and well-informed decisions can be made for the benefit of the communities.
\end{abstract}

Volume 5 Issue 2 - 2019

\author{
Pedro Bertemes Filho, John Alexander \\ Gómez-Sánchez \\ Department of Electrical Engineering, Universidade do Estado \\ de Santa Catarina (UDESC), Brazil

\begin{abstract}
Correspondence: Pedro Bertemes Filho, Department of (UDESC), Rua Paulo Malschitzki, 200 Zona Industrial Norte, Joinville / SC CEP: 89.219-7I0, Brazil, Tel +55 47 4009798I, Email pedro.bertemes@udesc.br
\end{abstract} \\ Electrical Engineering, Universidade do Estado de Santa Catarina
}

Received: March 22, 2019 | Published: April 22, 2019

\section{Background}

The development of biosensors based on physicochemical sensors has taken a significant step forward since 1960. These devices are used to measure physical variables in biological, industrial, and human processes. The main characteristic of biosensor is biological recognition of elements by using nanomaterials and biomimetic materials. The biosensor has high sensitivity and detection mechanisms through optical, electrical and piezoelectric properties. ${ }^{1}$ Some applications of biosensors include determination of physiological parameters, quality, and food safety, detection of contaminants in liquids, bacterial growth and discovery of metabolites in human body fluids. ${ }^{2}$

The detection of contaminants at low concentrations such as pesticides and other chemical elements in aqueous media using conventional techniques is low sensitive, requiring displacement of the sample to specialized laboratories and a long time for its analysis. ${ }^{3}$ The conventional electrochemical techniques, as the cyclic voltammetry, causes damaged in organic compounds for measurements of inorganic material in aqueous solutions. ${ }^{4}$ Chemicals compounds present in water with minimal quantities have a significant effect on public health, mainly caused by the accumulation and prolonged exposure to these products. Sometimes, conventional detection equipment of chemical contaminants in water has imprecision measurements in lower concentrations. ${ }^{5}$ The biosensors are considered as a powerful analytic tool. These devices have a faster time response, high sensitivity and selectivity for measurements in biological and chemical risk environments. ${ }^{6}$ The biosensors allow integration with electronic equipment to perform analyses in real time. ${ }^{7}$ The manufacture of biosensors to measure pollutants in aqueous media has some advantages as:

I. Easy synthesis in powder form. Allowing easy mounting on sensor elements.

II. Conformation of multiple substrates to obtain matrices of multiple contaminant sensors.

III. Versatility in the functionalization process to detect a specific type of pollutant.
IV. Implementation of high sensitivity and low-cost sensor systems for the detection of macromolecules by electrical impedance spectroscopy.

The biosensors are classified according to the heterogeneity of materials, their structural components according of chemical interaction, recognition element based on physical or physiological mechanisms, and the signal detection mechanisms using a transduction system. The biosensors can be based on screen printed electrodes (SPE) with a deposited layer of a polymer and nanoparticles. These substrates can have behavior as an Ion-sensitive field-effect transistors (ISFET). An electric response of biosensor changes with contaminants particles, here, the impedance measurements are affected with molecules over electrode substrate. ${ }^{9}$ An appropriate technique for each biosensor depends on the objective molecule, in some cases, the development process can be longer in time due to the non-stability process of the nanoparticle. ${ }^{10}$ Carbon-based compounds as graphite paste, graphene and nanotubes are used for the construction of biosensors and in pharmaceuticals samples with good results. ${ }^{11}$ Graphene biosensor has been used to detect glucose, cancer, antibodies, heavy metals, pathogenic bacteria combined with electric measurements. ${ }^{12}$ The biosensor devices have higher development on three lines of research: food, agriculture and human biological materials. The analysis of contaminants in water based on a biosensor requires detecting pesticides, antibiotics, and various microorganisms that affect health in human. ${ }^{13}$

Combinations of other measuring techniques together with biosensors have been used and reported in literature over the last decade. One of those technique is Electrical Impedance Spectroscopy (EIS), which is a non-destructive technique to analyze samples of any material solid and liquid including chemical composes and biological tissues. The EIS measurements in a frequency range use several electrodes geometries and covering and generating a characteristic spectrum from the material under test. The limitations of the technique correspond to processes of linearity, stability, and causality, depending on the precision of the instrumentation and experimental procedures. ${ }^{10}$ EIS was used to detect contaminants in water as Sodium Nitrate $\left(\mathrm{NaNO}_{3}\right)$, Ammonium Nitrate $\left(\mathrm{NH}_{4} \mathrm{NO}_{3}\right)^{14}$ and $\mathrm{f}$ di(2-ethylhexyl) phthalate (DEHP), ${ }^{15}$ bacterial (Listeria monocytogenes) ${ }^{16}$ with a higher correlation. 
EIS is a potential alternative compared to cyclic voltammetry methodologies, a non-destructive approach to the use of dilute products during the analysis procedures, guaranteeing high repetitiveness. ${ }^{17}$ Also, this technique can be used in situ applications due to low cost, portability and wireless circuits..$^{18}$ Limitation of biosensors depending on molecule used as a transducer. The regulation and activation mechanisms have restrictions, and they can generate similar responses. For this purpose, the biosensors require several laboratory tests to reduce uncertainly. ${ }^{19}$

\section{Conclusion}

A combined technique using a biosensor with EIS can reduce the technical limitations to characterize water quality in situ. The main advantages with this fusion is time reduction to obtain a data, a higher selectivity, specificity, portability, easy handling and low cost. The results allow to take decisions about safe and innocuous potable water treatment and irrigation to culture.

\section{Acknowledgments}

We thank the financial support of CAPES and UDESC for the institutional one

\section{Conflicts of interests}

Authors declare that there is no conflict of interest.

\section{References}

1. Lu M, Liu Y, Geng J, et al. Engineering nanomaterials-based biosensors for food safety detection. Biosens Bioelectron. 2018; 106:122-128.

2. Silva LMDC, Dos Santos VPS, Salgado AM, et al. Biosensors for Contaminants Monitoring in Food and Environment for Human and Environmental Health. In: Toonika Rinken, editor. State of the Art in Biosensors. IntechOpen; 2013:151-168.

3. Amarasekare KG, Shearer PW, Mills NJ. Testing the selectivity of pesticide effects on natural enemies in laboratory bioassays. Biological Control. 2016;102:7-16.

4. Scholz F. Voltammetric techniques of analysis: the essentials. Chem Texts. 2015;1:17.

5. Thomas SP, Sri Ranjan R, Webster GR, et al. Protocol for the Analysis of High Concentrations of Benzene, Toluene, Ethylbenzene, and Xylene Isomers in Water Using Automated Solid-Phase Microextraction-GCFID. Environmental Science \& Technology. 1996:30(5):1521-1526.

6. Gooding JJ. Biosensor technology for detecting biological warfare agents: Recent progress and future trends. Analytica Chimica Acta. 2006;559:137-151.
7. De la Cova C, Townley R, Regot S, et al. A Real-Time Biosensor for ERK Activity Reveals Signaling Dynamics during C. elegans Cell Fate Specification. Developmental Cell. 2017;42(5):542-553.

8. Vigneshvar S, Sudhakumari C, Senthilkumaran B, et al. Recent Advances in Biosensor Technology for Potential Applications - An Overview. Front Bioeng Biotechnol. 2016;4:11.

9. Pandey A, Gurbuz Y, Ozguz V, et al. Graphene-interfaced electrical biosensor for label-free and sensitive detection of foodborne pathogenic E. coli O157: H7. Biosensors and Bioelectronics. 2017;91:225-231.

10. Burcu Bahad E, Kemal Sezgintürk M. A review on impedimetric biosensors. Artificial Cells, Nanomedicine, and Biotechnology, Early Online. 2014:1-15.

11. Manjunatha P, Arthoba Nayaka Y, Chethana BK, et al. Development of multi-walled carbon nanotubes modified pencil graphite electrode for the electrochemical investigation of aceclofenac present in pharmaceutical and biological samples. Sensing and Bio-Sensing Research. 2018;17:717.

12. Kumar Krishnan S, Singh E, Pragya Singh et al. A review on graphenebased nanocomposites for electrochemical and fluorescent biosensors RSC Adv. 2019;9:8778-8881.

13. Ejeian F, Etedali P, Mansouri-Tehrani AH, et al. Biosensors for wastewater monitoring: A review. Biosensors and Bioelectronics. 2018;118:66-79.

14. Md Yunus MA, Mukhopadhyay SC. Planar Electromagnetic Sensor for the Detection of Nitrate and Contamination in Natural Water Sources Using Electrochemical Impedance Spectroscopy Approach. New Developments and Applications in Sensing Technology. 2011:39-63.

15. Zia AI, Mohd Syaifudin AR, Mukhopadhyay SC, et al. Electrochemical impedance spectroscopy based MEMS sensors for phthalates detection in water and juices. J Phys Conf Ser. 2013;439:012026.

16. Chen Q, Lin J, Gan C, et al. A sensitive impedance biosensor based on immunomagnetic separation and urease catalysis for rapid detection of Listeria monocytogenes using an immobilization-free interdigitated array microelectrode. Biosensors and Bioelectronics. 2015;74:504-511.

17. Tran AK, Sapkota A, Wen, J et al. Linear relationship between cytoplasm resistance and hemoglobin in red blood cell hemolysis by electrical impedance spectroscopy \& eight-parameter equivalent circuit. Biosensors and Bioelectronics. 2018;119:103-109.

18. Ghaffari SA, Caron WO, Loubier M et al. A Wireless Multi-Sensor Dielectric Impedance Spectroscopy Platform. Sensors. 2015; 15:2357223588 .

19. Carpenter AC, Paulsen IT, Williams TC. Blueprints for Biosensors: Design, Limitations, and Applications. Genes (Basel). 2018; 9(8):375. 